Abstract

\title{
Structural Basis for Capsid Recruitment and Coat Formation during HSV-1 Nuclear Egress ${ }^{\dagger}$
}

\author{
Elizabeth Draganova ${ }^{1}$, Jiayan Zhang ${ }^{2}$, Hong Zhou ${ }^{2}$ and Ekaterina Heldwein 1,* \\ 1 Department of Molecular Biology and Microbiology, Tufts University School of Medicine, Boston, \\ MA 02111, USA; elizabeth.draganova@tufts.edu \\ 2 Department of Microbiology, Immunology \& Molecular Genetics, University of California, Los Angeles, \\ CA 90095, USA; zhangjiayan@ucla.edu (J.Z.); Hong.Zhou@ucla.edu (H.Z.) \\ * Correspondence: katya.heldwein@tufts.edu \\ † Presented at Viruses 2020-Novel Concepts in Virology, Barcelona, Spain, 5-7 February 2020.
}

Published: 24 June 2020

\begin{abstract}
During herpesvirus infection, nascent viral capsids egress the nucleus into the cytoplasm by an unusual mechanism whereby capsids bud at the inner nuclear membrane. This process is mediated by the conserved heterodimeric nuclear egress complex (NEC), anchored to the inner nuclear membrane, that deforms the membrane around the capsid by forming a hexagonal array. However, how the NEC coat interacts with the capsid and how proper curvature of the coat is achieved to enable budding are yet unclear. Here, we show that the binding of a capsid protein, UL25, promotes the formation of a pentagonal rather than hexagonal NEC arrangement. Our results suggest that during nuclear budding interactions between the UL25 bound to the pentagonal capsid vertices and the NEC introduce pentagonal insertions into the hexagonal NEC array to yield an NEC coat of the appropriate size and curvature, leading to the productive budding and egress of UL25decorated capsids.
\end{abstract}

Keywords: herpes simplex virus type 1; herpesvirus; nuclear egress; nuclear egress complex; membrane budding; capsid budding

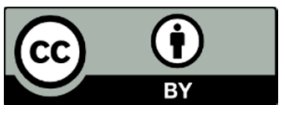

(c) 2020 by the authors. Licensee MDPI, Basel, Switzerland. This article is an open access article distributed under the terms and conditions of the Creative Commons Attribution (CC BY) license (http://creativecommons.org/licenses/by/4.0/). 